\title{
Newly Diagnosed Sarcopenia and Alzheimer's Disease in an Older Patient With Chronic Inflammation
}

\author{
Sun-Hyung Kim, Deok Su Sin, Jae-Young Lim \\ Department of Rehabilitation Medicine, Seoul National University Bundang Hospital, Seoul National University College of Medicine, \\ Seongnam, Korea
}

\author{
Corresponding Author: \\ Deok Su Sin, MD \\ https://orcid.org/0000-0001-5920-6925 \\ Department of Rehabilitation Medicine, \\ Seoul National University Bundang \\ Hospital, 82 Gumi-ro 173beon-gil, \\ Bundang-gu, Seongnam 13620, Korea \\ E-mail: plexion0202@gmail.com
}

Received: January 21, 2019

Revised: March 19, 2019

Accepted: March 20, 2019

\begin{abstract}
A 78-year-old man presented with the aggravation of weakness in the lower extremities, gait disturbance, and cognitive impairment. He was diagnosed with sarcopenia, distal sensorimotor polyneuropathy, and Alzheimer's disease. Low-grade chronic elevation of inflammatory markers was also revealed. We assumed that chronic low-grade inflammation with aging, also called "inflammaging," contributed to the development of multiple comorbidities. After multidisciplinary treatment and comprehensive rehabilitation, he could ambulate again with minimal to moderate assistance. Various age-related disorders should be suspected when older patients present with chronic low-grade inflammation. (Ann Geriatr Med Res 2019;23:38-41)
\end{abstract}

Key Words: Inflammation, Sarcopenia, Alzheimer disease

\section{INTRODUCTION}

Chronic low-grade inflammation refers to the elevation of inflammatory markers in the absence of overt infection that develops with aging; it is thought to accelerate aging and play a major role in the progression and pathogenesis of many age-related comorbidities. ${ }^{1)}$ Recent studies suggest that chronic low-grade inflammation affects muscle metabolism and is a key predictor of sarcopenia and physical function in older patients. ${ }^{2)}$ Moreover, it is thought to be associated with many other age-related diseases such as Alzheimer's disease. ${ }^{3-5)}$ Here, we report the case of our patient who had multiple comorbidities and presented with chronic low-grade inflammation.

\section{CASE REPORT}

A 78-year-old man complained of weakness in lower extremities and recurrent falls since 2011. Because of the weakness aggravating for 2 months, he spent nearly all time in bed-ridden state. He had experienced multiple episodes of acute limb ischemia since 2011 due to the recurrent bilateral peripheral arterial embolism in his lower extremities. In addition, his wife reported that he experienced marked memory impairment and intermittent disorientation. His past medical history included multiple burst and compression fractures of the lumbar vertebrae, chronic obstructive pulmonary disease, hypertension, and diabetes mellitus. After visiting our outpatient clinic, he was admitted to our inpatients unit for further evaluation and comprehensive rehabilitation.

Neurological examination showed decreased muscle strength in the lower extremities, with a Medical Research Council grade of $3 / 3$ on both sides. Bilateral hypoesthesia to vibration, pain, temperature, and light touch sensation was observed below the knee level, along with decreased joint proprioception. An electro-diagnostic examination revealed distal sensorimotor polyneuropathy involving the lower extremities, with axonal involvement (Tables 1 and 2).

Evaluation of ambulatory function showed gait imbalance and poor gait speed. Specifically, a functional ambulation category of 2, a score of 3 on the Berg balance scale, and 10-m walking time of $108.3 \mathrm{sec}$ were observed. Additionally, severe muscle wasting was reported and quantified as grip strength of $15.7 \mathrm{~kg}$ and $17.4 \mathrm{~kg}$ on the right and left sides, respectively, and a skeletal muscle index of $5.35 \mathrm{~kg} / \mathrm{m}^{2}$ measured by bioelectrical impedance analysis (Inbody S10; Biospace, Seoul, Korea). These results were consistent with the definition of sarcopenia. ${ }^{6}$ Initial blood tests revealed abnormally elevated levels of inflammatory markers, including a white blood cell count of $18.900 / \mu \mathrm{L}$, erythrocyte sedimentation rate of $39 \mathrm{~mm} /$ $\mathrm{h}$, and high-sensitivity C-reactive protein (hsCRP) level of $2.38 \mathrm{mg} / \mathrm{dL}$. In addition, review of the medical records revealed the chronic elevation of the white blood cell count, erythrocyte sedimentation rate, and hsCRP on blood test since 2007 (Table 3). He also had an elevated level of interleukin (IL)-6 (10.72 pg/mL). 
Table 1. Results of the nerve conduction study

\begin{tabular}{|c|c|c|c|c|}
\hline Motor and sensory & Latency (ms) & Amplitude (mV) & Velocity $(\mathrm{m} / \mathrm{s})$ & Response \\
\hline \multicolumn{5}{|l|}{ Motor } \\
\hline Rt common peroneal - TA & 5.99 & 2.1 & & \\
\hline Lt common peroneal - TA & 6.09 & 2.0 & & \\
\hline Rt common peroneal - EDB & & & & No \\
\hline Lt common peroneal - EDB & & & & No \\
\hline Rt tibial - AH & 4.22 & 1.7 & & \\
\hline Lt tibial - AH & 4.48 & 1.7 & & \\
\hline Rt median - APB & 3.49 & 7.8 & 52.4 & \\
\hline Rt ulnar - ADM & 2.97 & 6.3 & 55.5 & \\
\hline \multicolumn{5}{|l|}{ Sensory } \\
\hline Rt sural & & & & No \\
\hline Lt sural & & & & No \\
\hline Rt superficial peroneal & & & & No \\
\hline Lt superficial peroneal & & & & No \\
\hline Rt median & 3.13 & 20.2 & & \\
\hline Rt ulnar & 3.07 & 23.3 & & \\
\hline
\end{tabular}

TA, tibialis anterior; EDB, extensor digitorum brevis; AH, abductor halluces; APB, abductor pollicis brevis; ADM, abductor digiti minimi; Rt, right; Lt, left.

Table 2. Results of electromyography

\begin{tabular}{|c|c|c|c|c|}
\hline Muscle & Fib & PSW & Poly & Interference pattern \\
\hline Rt vastus medialis & None & None & & Reduced \\
\hline Lt vastus medialis & None & None & Normal/Increased & Reduced \\
\hline Rt tibialis anterior & None & None & & Full \\
\hline Lt tibialis anterior & None & None & & Reduced \\
\hline Rt peroneus longus & None & None & & Reduced \\
\hline Rt gastrocnemius (medial) & None & None & & Reduced \\
\hline Lt gastrocnemius (medial) & None & None & & Reduced \\
\hline Rt abductor hallucis & None & None & Increased & Discrete \\
\hline Rt biceps brachii & & & & Full \\
\hline Rt first dorsal interosseous & & & & Full \\
\hline
\end{tabular}

Fib, fibrillation potential; PSW, positive sharp wave; Poly, polyphasic motor unit action potential; Rt, right; Lt, left.

Table 3. Levels of high-sensitivity C-reactive protein (hsCRP) and erythrocyte sedimentation rate (ESR)

\begin{tabular}{ccc}
\hline Date & hsCRP $(\mathrm{mg} / \mathrm{dL})$ & ESR $(\mathrm{mm} / \mathrm{h})$ \\
\hline $2007-04-05$ & 5.86 & 35 \\
$2011-10-24$ & 6.27 & 44 \\
$2012-06-08$ & 2.58 & 55 \\
$2015-05-12$ & 5.22 & 10 \\
$2018-07-09$ & 2.38 & 39 \\
\hline
\end{tabular}

We consulted a neurology specialist to evaluate the patient for cognitive impairment. With neurological evaluation, the patient was diagnosed with Alzheimer's disease with depression, based on the severe level of multi-domain cognitive impairment combined with depression observed on the Seoul Neuropsychological Screening Battery-II and diffuse brain atrophy observed on brain magnetic resonance imaging (Fig. 1). After the diagnosis, donepezil was prescribed, as recommended by the neurologist.
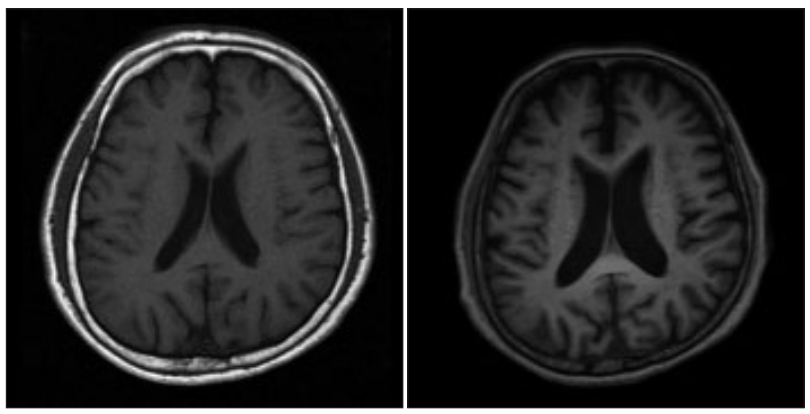

Fig. 1. Interval progression of brain atrophy: The above image shows coronal sections at the same level as that of the brain magnetic resonance imaging scan, performed in 2007 (left) and 2018 (right).

During admission, the patient received comprehensive physical therapy focusing on lower extremity strengthening and stepwise gait training daily. Anti-fall training and education for environmental modification were also 
provided to the patient and his caregiver to prevent further falls. We performed medication reconciliation for polypharmacy and potentially inappropriate medications for older adults ${ }^{7)}$ with the cooperation of multiple departments. At the time of discharge, he was able to ambulate with a walker for a maximum of 120 meters, and gait parameters all showed marked improvement (score on the Berg balance scale, 3 to 29; functional ambulation category, 2 to 3; 10-m walking time, 108.3 to $15.29 \mathrm{sec}$ ).

\section{DISCUSSION}

In this case, an older patient showed multiple disabling comorbidities that led him to a nearly bed-ridden state. A review of his past medical history revealed chronic elevation of inflammatory markers. Through a comprehensive and multidisciplinary approach, his physical function was enhanced to ambulatory levels at the time of discharge.

The patient's multiple disabilities met the operational criteria of frailty suggested by Fried and colleagues. ${ }^{8)} \mathrm{Ad}-$ ditionally, sarcopenia may have led the patient into the vicious cycle of frailty. ${ }^{90}$ However, unlike other typical frail older adults, signs of low-grade inflammation preceded the onset of frailty in this patient.

Some studies ${ }^{10-12)}$ reported epidemiologic evidence of the association between sarcopenia and elevated inflammatory markers. In these studies, the sarcopenic group showed high levels of hsCRP, IL-6, and tumor necrosis factor- $\alpha$. Although the exact mechanism is not fully understood yet, one animal study suggested that these inflammatory mediators affect muscle protein metabolism and consequently lead to muscle protein breakdown. ${ }^{13)}$

The association between chronic low-grade inflammation and Alzheimer's disease is also being widely studied. One theory explains this relationship as follows: proinflammatory cytokines such as interferon- $\gamma$ and IL-6 contribute to the production of peripheral amyloid-beta peptide. This might increase the concentration of amyloidbeta peptide in the brain, ${ }^{4)}$ which causes neuroinflammation and neuronal death. ${ }^{5)}$ Additionally, a recent study ${ }^{14)}$ showed that persistent upregulation of inflammatory cytokines in old mice hindered efficient peripheral nerve regeneration by affecting Schwann cell behavior and interfered with their differentiation after injury. These previous study results suggest the possible contribution of chronic low-grade inflammation to the patient's multiple comorbidities and abnormal aging.

In 2000, Franceschi et al. suggested the usage of the term "inflammaging" to describe the progressive sterile increase in proinflammatory status with aging. ${ }^{15)}$ Although "inflammaging" is only a theoretical mechanism, identifying it by assessing chronic elevation of inflammatory markers can be useful for earlier detection of multiple adverse health outcomes and earlier interventions. In our case, chronic elevation of inflammatory biomarkers was identified by reviewing medical records and made us rec- ognize the need to screen for comorbidities and to implement an intervention.

Currently, very little is known about chronic low-grade inflammation and its broad range of consequences. Its role in the aging process is also not clear. However, as in our case, it can coexist with multiple comorbidities, which is functionally devastating to patients. Therefore, early recognition of chronic low-grade inflammation in older patients and screening for related pathologies should be considered.

\section{CONFLICTS OF INTEREST DISCLOSURES}

The researchers claim no conflicts of interest.

\section{REFERENCES}

1. Wu IC, Lin CC, Hsiung CA. Emerging roles of frailty and inflammaging in risk assessment of age-related chronic diseases in older adults: the intersection between aging biology and personalized medicine. Biomedicine (Taipei) 2015;5:1.

2. Dalle S, Rossmeislova L, Koppo K. The role of inflammation in age-related sarcopenia. Front Physiol 2017;8:1045.

3. Sastre M, Dewachter I, Landreth GE, Willson TM, Klockgether T, van Leuven $\mathrm{F}$, et al. Nonsteroidal anti-inflammatory drugs and peroxisome proliferator-activated receptor-gamma agonists modulate immunostimulated processing of amyloid precursor protein through regulation of beta-secretase. J Neurosci 2003;23:9796804.

4. Blasko I, Marx F, Steiner E, Hartmann T, Grubeck-Loebenstein B. TNFalpha plus IFNgamma induce the production of Alzheimer beta-amyloid peptides and decrease the secretion of APPs. FASEB J 1999;13:63-8.

5. Heneka MT, Carson MJ, El Khoury J, Landreth GE, Brosseron F, Feinstein DL, et al. Neuroinflammation in Alzheimer's disease. Lancet Neurol 2015;14:388-405.

6. Jang HK. How to diagnose sarcopenia in Korean older adults? Ann Geriatr Med Res 2018;22:73-9.

7. Kim MY, Etherton-Beer C, Kim CB, Yoon JL, Ga H, Kim HC, et al. Development of a consensus list of potentially inappropriate medications for Korean older adults. Ann Geriatr Med Res 2018;22:121-9.

8. Fried LP, Tangen CM, Walston J, Newman AB, Hirsch C, Gottdiener J, et al. Frailty in older adults: evidence for a phenotype. J Gerontol A Biol Sci Med Sci 2001;56:M146-56.

9. Xue QL. The frailty syndrome: definition and natural history. Clin Geriatr Med 2011;27:1-15.

10. Bano G, Trevisan C, Carraro S, Solmi M, Luchini C, Stubbs B, et al. Inflammation and sarcopenia: a systematic review and metaanalysis. Maturitas 2017;96:10-5.

11. Batsis JA, Mackenzie TA, Jones JD, Lopez-Jimenez F, Bartels SJ. Sarcopenia, sarcopenic obesity and inflammation: results from the 1999-2004 National Health and Nutrition Examination Survey. Clin Nutr 2016;35:1472-83.

12. Hida T, Imagama S, Ando K, Kobayashi K, Muramoto A, Ito K, et al. Sarcopenia and physical function are associated with inflam- 
mation and arteriosclerosis in community-dwelling people: the Yakumo study. Mod Rheumatol 2018;28:345-50.

13. Frasca D, Blomberg BB. Inflammaging decreases adaptive and innate immune responses in mice and humans. Biogerontology 2016;17:7-19.

14. Büttner R, Schulz A, Reuter M, Akula AK, Mindos T, Carlstedt A, et al. Inflammaging impairs peripheral nerve maintenance and regeneration. Aging Cell 2018;17:e12833.

15. Franceschi C, Bonafè M, Valensin S, Olivieri F, De Luca M, Ottaviani E, et al. Inflamm-aging. An evolutionary perspective on immunosenescence. Ann N Y Acad Sci 2000;908:244-54. 